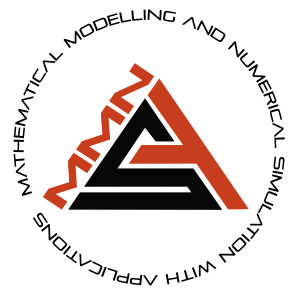

\title{
Numerical solutions and synchronization of a variable-order fractional chaotic system
}

\section{Zakia Hammouch 1,2,3,§, Mehmet Yavuz 4,5,§ and Necati Özdemir@6,*,§}

${ }^{1}$ Division of Applied Mathematics, Thu Dau Mot University, Binh Duong Province, Vietnam, ${ }^{2}$ Department of Medical Research, China Medical University Hospital, Taichung, Taiwan, ${ }^{3}$ Department of Sciences, École Normale Supérieure, Moulay Ismail University of Meknes, Morocco, ${ }^{4}$ Department of Mathematics and Computer Sciences, Necmettin Erbakan University, 42090 Konya, Turkey, ${ }^{5}$ Department of Mathematics, College of Engineering, Mathematics and Physical Sciences, University of Exeter, TR10, Cornwall, UK, ${ }^{6}$ Department of Mathematics, Balikesir University, 10440 Balikesir, Turkey

*Corresponding Author

§hammouch_zakia@tdmu.edu.vn (Zakia Hammouch); mehmetyavuz@erbakan.edu.tr (Mehmet Yavuz); nozdemir@balikesir.edu.tr (Necati Özdemir)

\begin{abstract}
In the present paper, we implement a novel numerical method for solving differential equations with fractional variableorder in the Caputo sense to research the dynamics of a circulant Halvorsen system. Control laws are derived analytically to make synchronization of two identical commensurate Halvorsen systems with fractional variable-order time derivatives. The chaotic dynamics of the Halvorsen system with variable-order fractional derivatives are investigated and the identical synchronization between two systems is achieved. Moreover, graph simulations are provided to validate the theoretical analysis.
\end{abstract}

Key words: Variable-order fractional derivative; chaotic system; Lyapunov exponent; synchronization AMS 2020 Classification: 34D06; 26A33; 34C28

\section{Introduction}

Recently chaos theory has attracted the scientific community. It has revalorized the evolution of science and technology immediately its appearance in 1963 [1]. This is primarily due to the unpredictable dynamic behavior and the sensitivity to initial conditions. The concept of chaotic science is extensively referred to the science of revelations, of the unpredictable and nonlinear. Therefore, when studying chaotic phenomena one should expect the unexpected. Besides, chaos theory has become an effective research area, because of the various applications of chaos in several disciplines like economy, chemistry, physics, engineering, ecology, robotics, secure communications etc [2]. In the literature, there are many familiar chaotic systems like: Lorenz system, Lu system, Ikeda system, Sprott-Linz system, Jerk system etc $[3,4,5,6,7,8,9,10,11,12,13,14,15,16,17,18,19,20,21,22,23,24,25,26,27,28$, $29,30,31]$.

Moreover, the modeling of problems in physics, engineering, and real-life phenomena reflects the mathematical tools available at the time of their development. Therefore, most real-life problems have been described by means of differential equations with non-integer order derivatives [32]. Recently, many papers focused their attention on ODEs and PDEs with non-integer-order derivatives owing to their common aspect in assorted applications in finance, medical, fluid mechanics, viscoelasticity, biology, physics, and engineering [33, 34, 35, 36, 37]. Therefore, there is abundant literature developed touching the applications of fractional differential equations in non-linear dynamics [38, 39, 40, 41, 42, 43, 44]. Accordingly, considerable attention of fractional

> Received: 13.07.2021 > Revised: 14.08.2021 > Accepted: 17.08.2021 > Published: 21.08.2021 
equations and their solutions have been given $[45,46,47,48,49,50,51,52,53,54,55,56,57,58,59,60,61]$. Nowadays, the variable-order fractional calculus (VOFC) is becoming a very useful instrument, due to the numerous applications in science and engineering [62] and a few studies have been declared in the literature using derivatives with variable-order [63, 64]. More recently, in [65], a physical empirical study that includes the variable-order operators has been investigated.

In this paper, we will discuss a novel numerical method for obtaining the solutions of ODEs with variable-order time-fractional derivatives (VOFD). We then study the chaotic dynamics of a Halvorsen system with VOFD and achieve the identical synchronization between two systems.

Our paper organization is as follows: Section 2 deals with some fundamental definitions of VOFC and stability theory as well as it introduces a new numerical scheme for solving fractional-ordered DEs. Besides three illustrative examples explain the comparisons between solutions we obtained and the results in the literature in this section. In section 3, a circulant chaotic system with fractional-order derivatives is presented, its qualitative properties are explained in detail. Section 4 deals with the synchronization results. In section 5, numerical simulations are reported. Finally, in section 6, the main conclusions are outlined.

\section{Preliminaries}

\section{Preliminaries for variable-order fractional calculus}

In this section, we recall some definitions and properties of the VOFC; they are obtained by changing the order of the fractional derivation by a continuous bounded function in the counterparts.

Definition 1 [66] For any bounded function $\mathrm{k}(t)$, the variable-order Caputo fractional derivative (VOCFD) of a function $\phi$ is given by

$$
D_{C}^{\kappa(t)} \phi(t)=\frac{1}{\Gamma(r-\kappa(t))} \int_{0}^{t}\left[\frac{\phi^{(r)}(s)}{(t-s)^{\kappa(s)+1-r}}\right] d s,
$$

as long as the integral exists, with $r-1<\mathrm{k}(t) \leq r, r=\left[\max _{0 \leq t \leq T} \mathrm{k}(t)\right]+1$, where $[\rho]$ is the integer part of $\rho$, and $\Gamma($.$) is the Gamma function.$ When $\mathrm{k}(t)$ is a constant, then we retrieve the constant-order fractional derivative in the Caputo sense.

Remark 1 Throughout this paper we think that the function $\kappa(t)$ is defined such that the integral in the previous definition exists.

Remark 2 Theoretical analysis on existence of solutions of various initial value problems with VOFDs has been given in some studies (see for instance [62] and [67]).

\section{The stability theorem}

Consider a general variable-order fractional (VOF) system

$$
\left\{\begin{array}{l}
D_{c}^{\kappa(t)} x(t)=\xi_{1}(x, y, z), \\
D_{c}^{\kappa(t)} y(t)=\xi_{2}(x, y, z), \\
D_{c}^{\kappa(t)} z(t)=\xi_{3}(x, y, z),
\end{array}\right.
$$

where $\kappa(t) \in(0,1]$ is the order function that is bounded and continuous, $t \geq 0$, and initial conditions $(x(0), y(0), z(0))=\left(x_{0}, y_{0}, z_{0}\right)$. The equilibrium of system (2) can be deduced via solving the coupled equations

$$
\left\{\begin{array}{l}
\xi_{1}(x, y, z)=0, \\
\xi_{2}(x, y, z)=0, \\
\xi_{3}(x, y, z)=0,
\end{array}\right.
$$

and the Jacobian of system (2) is shown as follows

$$
J=\left[\begin{array}{lll}
\frac{\partial \xi_{1}}{\partial x} & \frac{\partial \xi_{1}}{\partial y} & \frac{\partial \xi_{1}}{\partial z} \\
\frac{\partial \xi_{2}}{\partial x} & \frac{\partial \xi_{2}}{\partial y} & \frac{\partial \xi_{2}}{\partial z} \\
\frac{\partial \xi_{3}}{\partial x} & \frac{\partial \xi_{3}}{\partial y} & \frac{\partial \xi_{3}}{\partial z}
\end{array}\right]
$$

The stability of system (2) counts on the stability of eigenvalues $\lambda_{i}$ of the Jacobian J. To categorize the equilibrium point of system (2), we will use the extended necessary stability condition for VOF systems [66].

We denote $\kappa_{R}=\max _{0 \leq t \leq T} \kappa(t)$ and $\kappa_{r}=\min _{0 \leq t \leq T} \kappa(t)$.

Theorem 1 Say that $E$ is a given equilibrium point of the following autonomous system

$$
D_{t}^{\kappa(t)} X(t)=F(X),
$$


where $X(0)=X_{0}, 0<\kappa(t) \leq 1$ is bounded and continuous and $X \in \mathbb{R}^{n}$.

If the $\lambda_{i}$ values of $J=\left.\frac{\partial F}{\partial X}\right|_{E}$ hold

$$
\left|\arg \left(\lambda_{i}\right)\right|>\frac{\pi}{2} \kappa_{R}
$$

in that case system (5) is locally asymptotically stable at the balance value E. Else, if $\left|\arg \left(\lambda_{i}\right)\right|<\frac{\pi}{2} \kappa_{r}$ system (5) is unstable.

\section{A numerical scheme for solving VOF differential equations}

Taking into account that variable-order fractional differentiation is a generalization constant-order fractional differentiation (COFD), some well-known relations including composition and sequential derivative rules for COFD do not remain valid for VOFD. Consequently, solving differential equations under variable-order derivatives needs different methodologies, modifications, and/or generalizations for the known concepts. Inspired by the recent works [68] and [69], we introduce in what follows a new scheme for solving FDEs with variable-order.

Let us take the following VOF system:

$$
\left\{\begin{array}{l}
{ }_{0}^{C} D x^{\kappa}(t)(t)=F(t, \chi(t)) \text { for } 0<t \leq T \\
\chi(0)=x_{0}
\end{array}\right.
$$

where $F$ is a general nonlinear function, $0<\kappa(t) \leq 1$ and $x_{0}$ is the initial condition. Applying the operator $I^{\kappa(t)}$ on both sides of equation (7) we get

$$
\chi(t)=x_{0}+\frac{1}{\Gamma(\kappa(t))} \int_{0}^{t} F(s, x(s))(t-s)^{\kappa(t)-1} d s .
$$

Now we choose the following uniform grid:

$$
h=\frac{T}{N}, t_{n}=n h, \text { for } n=0,1,2, \ldots, N, t_{0}=0 \text { and } T_{n}=T .
$$

For a given $t=t_{n+1}, \quad n=0,1,2 \ldots, N$ it yields

$$
\begin{aligned}
x\left(t_{n+1}\right) & =x_{0}+\frac{1}{\Gamma(\kappa(t))} \int_{0}^{t_{n+1}} F(s, x(s))\left(t_{n+1}-s\right)^{\kappa(t)-1} d s \\
& =x_{0}+\frac{1}{\Gamma(\kappa(t))} \sum_{k=0}^{n} \int_{t_{k}}^{t_{k+1}} F(s, x(s))\left(t_{n+1}-s\right)^{\kappa(t)-1} d s .
\end{aligned}
$$

It is well-known that composite Lagrange interpolation consists in splitting the interval into many subintervals, and uses a lower order Lagrange interpolation in each subinterval, in order to have a good approximation of a function. Therefore, on each subinterval $\left[t_{k}, t_{k+1}\right]$, we approximate $F(s, x(s))$ with a Lagrange interpolation polynomial:

$$
\begin{aligned}
P_{k}(\tau) & =\frac{s-t_{k-1}}{t_{k}-t_{k-1}} F\left(t_{k}, x\left(t_{k}\right)\right)-\frac{s-t_{k}}{t_{k}-t_{k-1}} F\left(t_{k-1}, x\left(t_{k-1}\right)\right) \\
& =\frac{F\left(t_{k}, x\left(t_{k}\right)\right)}{h}\left(s-t_{k-1}\right)-\frac{F\left(t_{k-1}, x\left(t_{k-1}\right)\right)}{h}\left(s-t_{k}\right) \\
& \simeq \frac{F\left(t_{k}, x_{k}\right)}{h}\left(s-t_{k-1}\right)-\frac{F\left(t_{k-1}, x_{k-1}\right)}{h}\left(s-t_{k}\right) .
\end{aligned}
$$

Coming back to (9), we get the following

$$
\begin{aligned}
x_{n+1}= & x_{0}+\frac{1}{\Gamma(\kappa(t))} \sum_{k=0}^{n} \frac{F\left(t_{k}, x_{k}\right)}{h} \int_{t_{k}}^{t_{k+1}}\left(s-t_{k-1}\right)\left(t_{n+1}-s\right)^{\kappa(t)-1} d s \\
& -\frac{1}{\Gamma(\kappa(t))} \sum_{k=0}^{n} \frac{F\left(t_{k-1}, x_{k-1}\right)}{h} \int_{t_{k}}^{t_{k+1}}\left(s-t_{k}\right)\left(t_{n+1}-s\right)^{k(t)-1} d s .
\end{aligned}
$$

Next, we compute the following coefficients

$$
\mathcal{A}_{\mathrm{k}(t), k, 1}=\int_{t_{k}}^{t_{k+1}}\left(s-t_{k-1}\right)\left(t_{n+1}-s\right)^{\mathrm{K}(t)-1} d s
$$

and

$$
\mathcal{B}_{\kappa}(t), k, 2=\int_{t_{k}}^{t_{k+1}}\left(s-t_{k}\right)\left(t_{n+1}-s\right)^{\kappa(t)-1} d s
$$


A simple integration leads to

$$
\mathcal{A}_{\kappa}(t), k, 1=\frac{(n+1-k)^{\kappa(t)}(n-k+2+\kappa(t))-(n-k)^{\kappa(t)}(n-k+2+2 \kappa(t))}{\kappa(t)(\kappa(t)+1)},
$$

and

$$
\mathcal{B}_{\kappa(t), k, 2}=\frac{(n+1-k)^{\kappa(t)+1}-(n-k)^{\kappa(t)}(n-k+1+\kappa(t))}{\kappa(t)(\kappa(t)+1)} .
$$

Inserting (11) and (12) in equation (10) gives the following approximation

$$
\begin{aligned}
x_{n+1}= & x_{0}+\sum_{k=0}^{n} Q_{k}\left((n+1-k)^{\kappa(t)}(n-k+2+\kappa(t))-(n-k)^{\kappa(t)}(n-k+2+2 \kappa(t))\right) \\
& -\sum_{k=0}^{n} Q_{k-1}\left((n+1-k)^{\kappa(t)+1}-(n-k)^{\kappa(t)}(n-k+1+\kappa(t))\right) .
\end{aligned}
$$

$Q_{k}=\frac{h^{\kappa(t)} F\left(t_{k}, x_{k}\right)}{\Gamma(\kappa(t)+2)}$ and $Q_{k-1}=\frac{h^{\kappa(t)} F\left(t_{k-1}, x_{k-1}\right)}{\Gamma(\kappa(t)+2)}$.

To prove the accuracy and the applicability of the above described method, we give some examples and find their solutions. The obtained numerical solutions are compared with the exact solutions if the case arises, otherwise, we made a comparison with obtained results via other known methods.

Example 1 First we take the following linear FDE, where the fractional operator is taken in the Caputo sense:

$$
\left\{\begin{array}{l}
D_{c}^{\kappa(t)} \chi(t)=\cos (2 t), \quad t \in[0, T] \\
x(0)=0 .
\end{array}\right.
$$

The application of the fractional integral on both sides of (14) gives the following exact solution:

$$
\begin{aligned}
x(t)= & \frac{-2^{3 / 2-\kappa(t)} t \alpha(t) \mathbf{L S}(\kappa(t)+1 / 2,3 / 2,2 t)+2 t^{\kappa(t)+1 / 2} \kappa(t)}{2 \Gamma(2+\kappa(t)) \sqrt{t}} \\
& +\frac{2 t^{\kappa(t)+1 / 2}-2^{-\kappa(t)+1 / 2} \mathbf{L S}(\kappa(t)+3 / 2,1 / 2,2 t)}{2 \Gamma(2+\kappa(t)) \sqrt{t}},
\end{aligned}
$$

where $\mathbf{L S}$ is the Lommel's function. Let us take $\mathrm{k}(t)=0.9-0.05 \frac{t}{1+t}$ solve equation (14) numerically using the above proposed scheme for a step-size $h=0.01, N=1000$ and $T=10$. Figure 1 plots the profile of numerical solution vs exact solution of (14), it is clear that the suggested algorithm furnishes accurate numerical results.

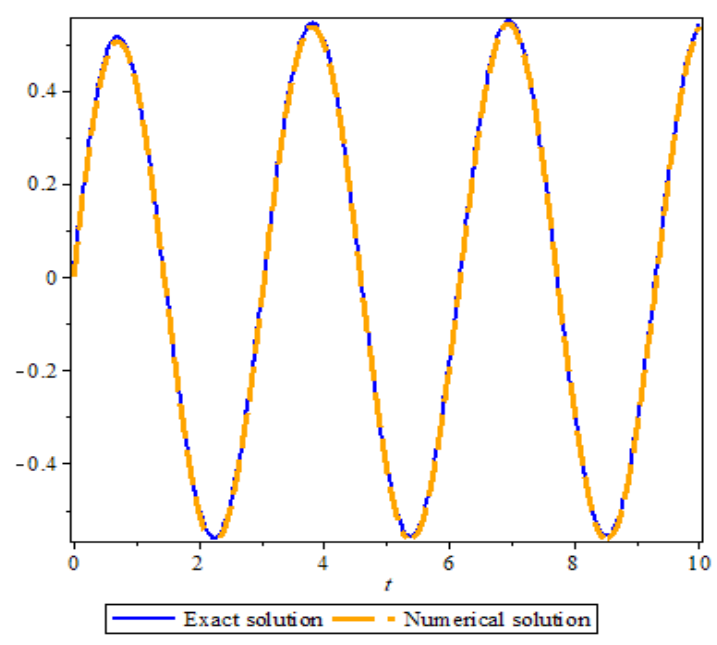

Figure 1. Exact vs numerical solution of $(14)$ for $k(t)=0.9-0.05 \frac{t}{1+t}$. 
Example 2 Now we take into account the following equation

$$
\left\{\begin{array}{l}
D_{c}^{\kappa(t)} x(t)=\mathrm{e}^{-\sqrt{t^{2}}}, \quad t \in[0, T] \\
x(0)=0
\end{array}\right.
$$

Similarly, we get the following exact solution for equation (15)

$$
\begin{aligned}
x(t)= & \frac{t^{1 / 2 \kappa(t)} \mathrm{e}^{-1 / 2 t} \mathrm{~W}(-1 / 2 \kappa(t), 1 / 2 \kappa(t)+1 / 2, t)}{t(1+\kappa(t)) \Gamma(\kappa(t))} \\
& +\frac{t^{1 / 2 \kappa(t)} \mathrm{e}^{-1 / 2 t} \mathbf{W}(-1 / 2 \kappa(t)+1,1 / 2 \kappa(t)+1 / 2, t)}{t \kappa(t)(1+\kappa(t)) \Gamma(\kappa(t))},
\end{aligned}
$$

where $\mathbf{W}$ is the Whittaker function.

To prove the high accuracy of the novel method, we solve equation (15) by taking $k(t)=0.94+\frac{1}{30} \sin \left(\frac{t}{6}\right), h=0.05$ and $T=10$. Figure 2 shows that an excellent agreement between the exact and numerical solution of (15).

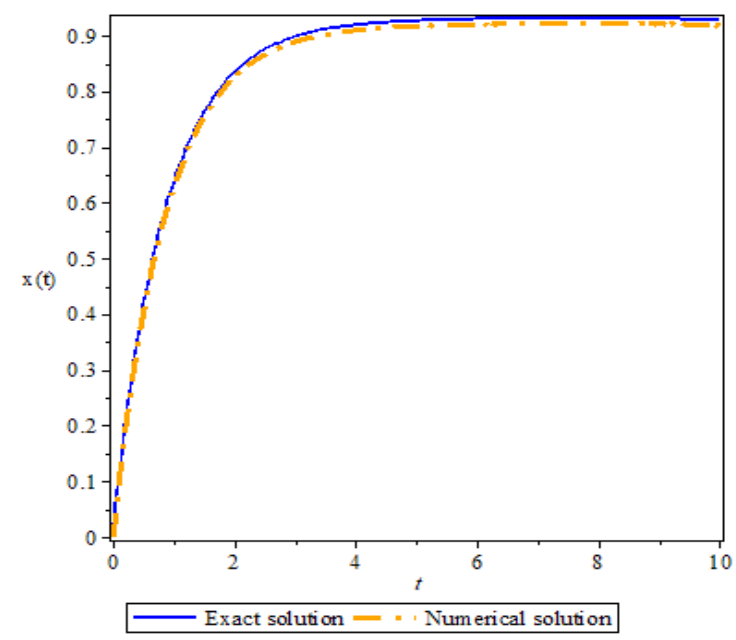

Figure 2. Exact vs numerical solution of (15) for $k(t)=0.94+\frac{1}{30} \sin \left(\frac{t}{6}\right)$

Example 3 Let us now consider the problem for the VFO Duffing oscillator [32]

$$
\left\{\begin{array}{l}
x^{\prime \prime}(t)+0.2 D_{c}^{\kappa(t)} x(t)+x(t)+x^{3}(t)=p(t) \\
x(0)=0, x^{\prime}(0)=0
\end{array}\right.
$$

where

$$
\kappa(t)=1-\exp (-t), \text { and } p(t)=2+t^{2}+t^{6}+0.4 \frac{t^{1+\mathrm{e}^{-t}} \mathrm{e}^{t}}{\Gamma\left(\mathrm{e}^{-t}\right)\left(1+\mathrm{e}^{-t}\right)}
$$

The exact solution of Eq. (16) is $x(t)=t^{2}$. In Figure 3, we remark that the solution we obtained and the exact solution are in high agreement for $h=0.01$. 


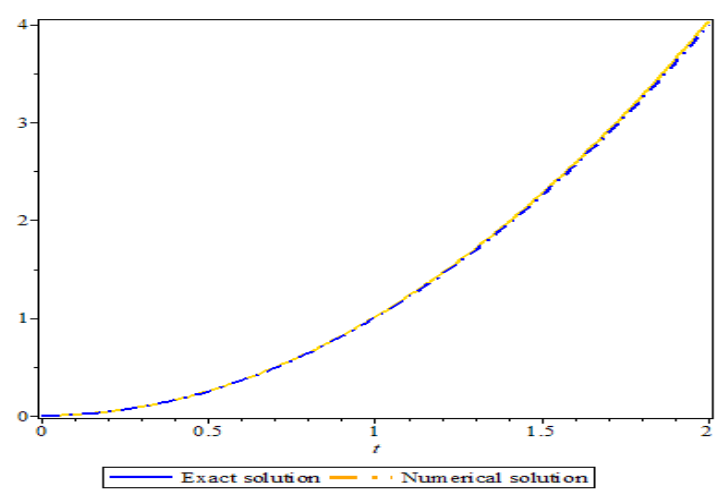

Figure 3. Exact vs numerical solution of $(16)$ for $\kappa(t)=1-\exp (-t)$.

\section{A variable-order fractional Halvorsen system}

This section aims to present a study of a 3D circulant system, called the Halvorsen system [3] with variable fractional-order derivative in Caputo sense, which is described by the following

$$
\left\{\begin{array}{l}
D_{c}^{q(t)} x=-a x-b y-b z-y^{2}, \\
D_{c}^{q(t)} y=-a y-b z-b x-z^{2}, \\
D_{c}^{q(t)} z=-a z-b x-b y-x^{2} .
\end{array}\right.
$$

It is clear that system (18) is symmetric respectively to cyclic interchanges of the states $x, y$, and $z$. According to Halvorsen, system (18) is chaotic for the values of the parameters are given as $a=1.3$ and $b=4$ (for the classical case $q(t)=1$ ). In what follows, we describe the qualitative properties of the Halvorsen chaotic system (18). Throughout this section we will take $q(t)=$ $0.7+0.2 \frac{\exp (-t)}{1+\exp (-t)}$.

\section{Dissipativity}

As in [3], the Halvorsen system (18) can be written as

$$
D_{C}^{q(t)} X(t)=F(X(t))
$$

where $X=\left[\begin{array}{c}x \\ y \\ z\end{array}\right], F=\left[\begin{array}{c}f_{1}(X) \\ f_{2}(X) \\ f_{3}(X)\end{array}\right]$ and

$$
\left\{\begin{array}{l}
f_{1}(X)=-a x-b y-b z-y^{2}, \\
f_{2}(X)=-a y-b z-b x-z^{2}, \\
f_{3}(X)=-a z-b x-b y-x^{2} .
\end{array}\right.
$$

The divergence of the vector field $f$ on $\mathbb{R}^{3}$ is expressed as

$$
\operatorname{div} F=\frac{\partial f_{1}}{\partial x}+\frac{\partial f_{2}}{\partial y}+\frac{\partial f_{3}}{\partial z}=-3.9<0 .
$$

Let us denote by $\Omega$ a subset of $\mathbb{R}^{3}$ with a smooth boundary such that $\Omega(t)=\Phi_{t}(\Omega)$ where $\Phi_{t}$ is the flow of $F$. Additionally, let $V(t)$ refers to the hypervolume of $\Omega(t)$. By the Liouville's theorem, we get

$$
\frac{d V}{d t}=\int_{\Omega(t)} \operatorname{div} F d x d y d z
$$

Replacing divF from (20) into (21), we get

$$
\frac{d V}{d t}=-3.9 \int_{\Omega(t)} d x d y d z=-3.9 V(t)
$$


Integrating equation (22) we obtain

$$
V(t)=V(0) \exp (-3.9 t)
$$

According to Eq. (23), $V(t)$ is converging to zero exponentially as $t$ becomes infinite. Consequently, the VOF Halvorsen system (18) is a dissipative one.

\section{Equilibrium point and the stability}

The equilibria of the VOF Halvorsen system (18) are deduced by solving the following system

$$
\left\{\begin{array}{l}
-a x-b y-b z-y^{2}=0, \\
-a y-b z-b x-z^{2}=0, \\
-a z-b x-b y-x^{2}=0 .
\end{array}\right.
$$

We find that (24) has two equilibrium points, namely

$$
E_{0}=(0,0,0) \text { and } E_{1}=(-9.27,-9.27,-9.27) \text {. }
$$

The Jacobian matrix of the VOF Halvorsen system (24) at $E_{0}$ is obtained as

$$
J_{E_{0}}=\left[\begin{array}{ccc}
-1.27 & -4 & -4 \\
-4 & -1.27 & -4 \\
-4 & -4 & -1.27
\end{array}\right]
$$

The matrix $J_{E_{0}}$ has the eigenvalues

$$
\left[\begin{array}{c}
\lambda_{1}=2.73 \\
\lambda_{2}=-9.27 \\
\lambda_{3}=2.73
\end{array}\right]
$$

Similarly, the Jacobian matrix of system (24) at $E_{1}$ is given as

$$
J_{E_{1}}=\left[\begin{array}{ccc}
-1.27 & 14.54 & -4 \\
-4 & -1.27 & 14.54 \\
14.54 & -4 & -1.27
\end{array}\right]
$$

The eigenvalues of $J_{E_{1}}$ are :

$$
\left[\begin{array}{c}
\kappa_{1}=-6.54+16.0561109861635 i, \\
\kappa_{2}=-6.54-16.0561109861635 i, \\
\kappa_{3}=9.27
\end{array}\right]
$$

We conclude that the equilibrium point $E_{0}$ is a saddle then it is unstable. Therefore, the necessary condition to ensure chaos is satisfied.

\section{Quantitative characterization of VOFD Halvorsen system}

The computation of Lyapunov exponents (LE) is a basic problem in the study of dynamical systems since they provide a quantification of the exponential divergence of initially close state-space trajectories and measure the amount of chaos in a given system [3]. Actually, a positive (LE) is sufficient to claim the presence of chaos in a dynamical system.

A numerical calculation using the Gram-Schmidt orthonormalization procedure for the initial conditions $(x, y, z)=(0.2,0.6,0.2)$ reveals that system $(24)$ when $q(t)=0.7+0.2 \frac{\exp (-t)}{1+\exp (-t)}$, has the following Lyapunov exponents :

$$
\left[\begin{array}{c}
L_{1}=0.7935801 \\
L_{2}=0.0002090 \\
L_{3}=-4.6037936
\end{array}\right]
$$


Since $L_{1}+L_{2}+L_{3}=-3.8100045<0$, the VOF Halvorsen chaotic system (24) is dissipative.

Moreover, the Kaplan-Yorke dimension of the VOF Halvorsen chaotic system (24) is obtained as

$$
D_{K Y}=2+\frac{L_{1}+L_{2}}{\left|L_{3}\right|}=2.1724206533 \text {, }
$$

which is fractional.

\section{Active control synchronization}

The synchronization of two coupled chaotic systems is an important topic due to its applications in various fields of science and engineering, for instance, secure communication, cryptography, analog and digital signals, control processing, time series analysis, as well as earthquake dynamics [70]. Moreover, numerous techniques have been investigated for chaos synchronization like linear and nonlinear feedback control [71], back stepping nonlinear control approach [72], sliding mode control [73], adaptive control [74], etc. In this paper, we will design active nonlinear controllers to synchronize two identical Halvorsen systems with variable-order time-fractional derivatives.

To achieve synchronization, we define the drive-response scheme of two VOF identical Halvorsen systems, namely

$$
\begin{gathered}
\text { Drive }\left\{\begin{array}{l}
D_{t}^{q(t)} x_{1}=-a x_{1}-b y_{1}-b z_{1}-y_{1}^{2}, \\
D_{t}^{q(t)} y_{1}=-a y_{1}-b z_{1}-b x_{1}-z_{1}^{2}, \\
D_{t}^{q(t)} z_{1}=-a z_{1}-b x_{1}-b y_{1}-x_{1}^{2} .
\end{array}\right. \\
\text { Response }\left\{\begin{array}{l}
D_{t}^{q(t)} x_{2}=-a x_{2}-b y_{2}-b z_{2}-y_{2}^{2}+U_{1}(t), \\
D_{t}^{q(t)} y_{2}=-a y_{2}-b z_{2}-b x_{2}-z_{2}^{2}+U_{2}(t), \\
D_{t}^{q(t)} z_{2}=-a z_{2}-b x_{2}-b y_{2}-x_{2}^{2}+U_{3}(t),
\end{array}\right.
\end{gathered}
$$

where $U_{i}(t) ; i=1,2,3$ are unknown active control functions to be computer lated. Recall that the initial conditions $\left(x_{1,0}, y_{1,0}, z_{1,0}\right)$ and $\left(x_{2,0}, y_{2,0}, z_{2,0}\right)$ are different and we target to synchronize the signals even if there is discrepancy between the initial conditions. First, we define the error vector $\mathbf{e}(t)$ as the following

$$
\left\{\begin{array}{l}
e_{1}=x_{2}-x_{1} \\
e_{2}=y_{2}-y_{1} \\
e_{3}=z_{2}-z_{1}
\end{array}\right.
$$

Subtracting (32) from (33) and using (34), we find

$$
\left\{\begin{array}{l}
D_{t}^{q(t)} e_{1}=-a e_{1}-b e_{2}-b e_{3}-\left(y_{2}^{2}-y_{1}^{2}\right)+U_{1}(t), \\
D_{t}^{q(t)} e_{2}=-a e_{2}-b e_{3}-b e_{1}-\left(z_{2}^{2}-z_{1}^{2}\right)+U_{2}(t), \\
D_{t}^{q(t)} e_{3}=-a e_{3}-b e_{1}-b e_{2}-\left(x_{2}^{2}-x_{1}^{2}\right)+U_{3}(t) .
\end{array}\right.
$$

Let

$$
\left\{\begin{array}{l}
U_{1}(t)=b e_{2}+b e_{3}+\left(y_{2}^{2}-y_{1}^{2}\right) \\
U_{2}(t)=\left(z_{2}^{2}-z_{1}^{2}\right)+b e_{3} \\
U_{3}(t)=\left(x_{2}^{2}-x_{1}^{2}\right)
\end{array}\right.
$$

Consequently, the fractional-order error dynamical system is reduced to

$$
\left\{\begin{array}{l}
D_{t}^{q(t)} e_{1}=-a e_{1}, \\
D_{t}^{q(t)} e_{2}=-a e_{2}-b e_{1}, \\
D_{t}^{q(t)} e_{2}=-b e_{1}-b e_{2}-a e_{3} .
\end{array}\right.
$$


Theorem 2 For any initial conditions, the drive and response defined by the synchronization scheme (32) and (33) are with the control law (36).

Proof The above error system (37) has a unique equilibrium point $(0,0,0)$ and the Jacobian matrix at this point is

$$
J_{(0,0,0)}=\left[\begin{array}{ccc}
-1.3 & 0 & 0 \\
-4 & -1.3 & -4 \\
0 & 0 & -1.3
\end{array}\right]
$$

Clearly, $\lambda=-1.3$ is triple eigenvalue of $J_{(0,0,0)}$ and $|\arg (\lambda)|=\pi$ which is always greater than $\frac{\pi}{2} q_{M}$. Therefore, based on the stability theorem, we conclude that it is direct to see that the error dynamics converge to the manifold $\left(e_{1}, e_{2}, e_{3}\right)=(0,0,0)$ as $t \rightarrow \infty$. Consequently, the synchronization between two identical systems (32) and (33) is achieved via the control law (36).

\section{Numerical simulations}

This section presents the numerical simulations of Sections 3 and 4 . The time step is fixed to $h=0.01$ and the calculations are carried out for $q(t)=0.7+0.2 \frac{\exp (-t)}{1+\exp (-t)}$.
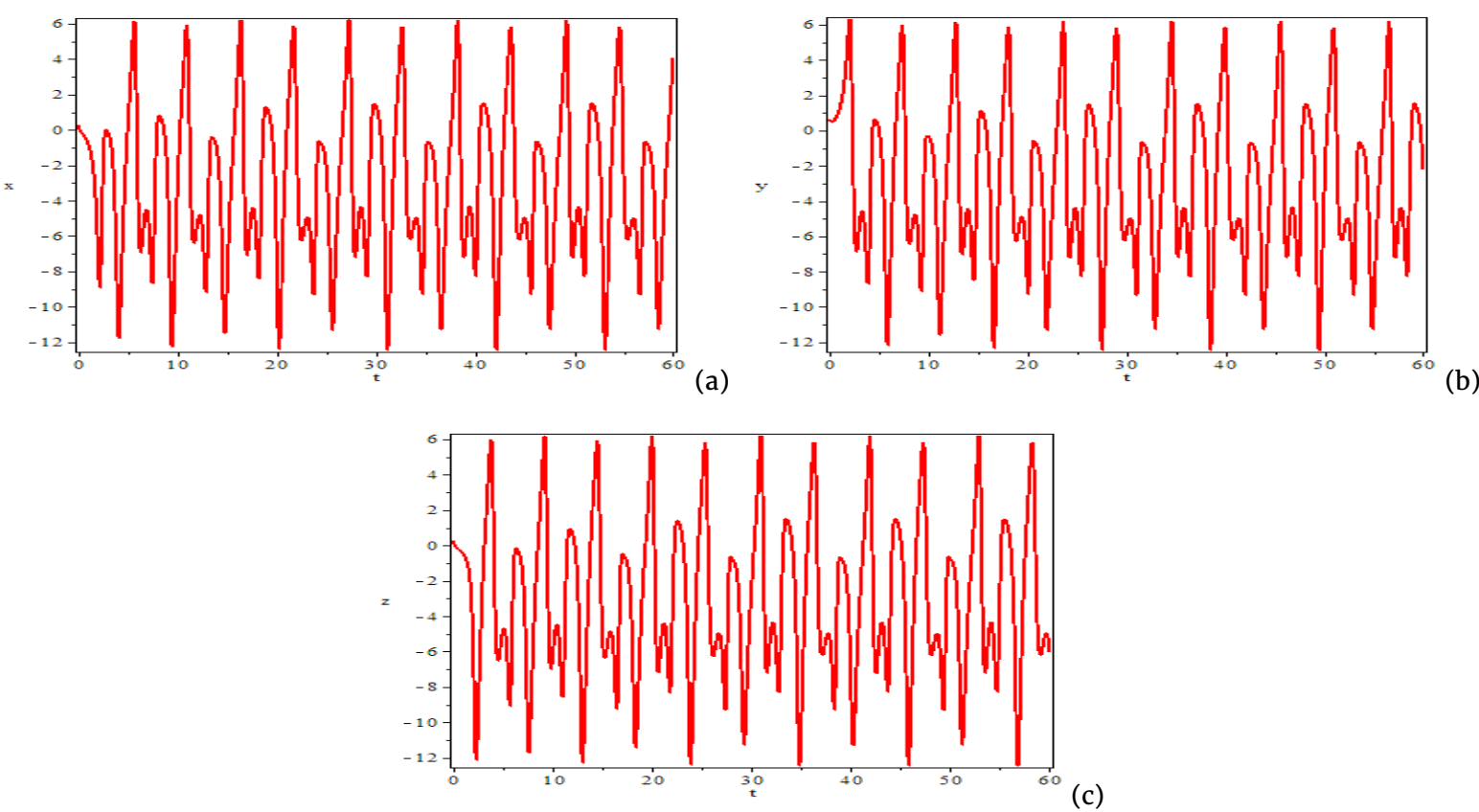

Figure 4. Time series of system (18): (a) $x(t)$, (b) $y(t)$ and (c) $z(t)$. 

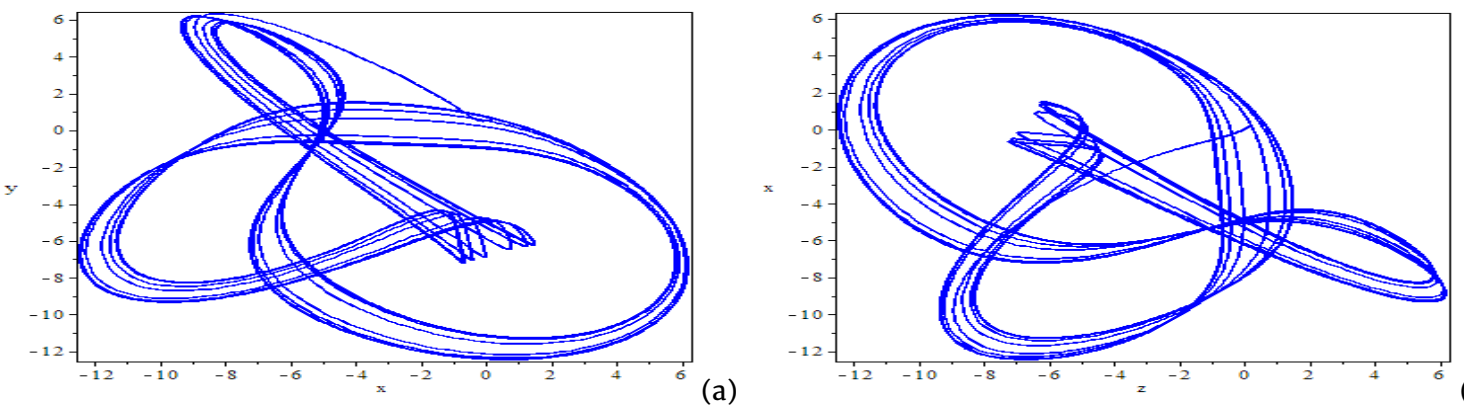

(b)
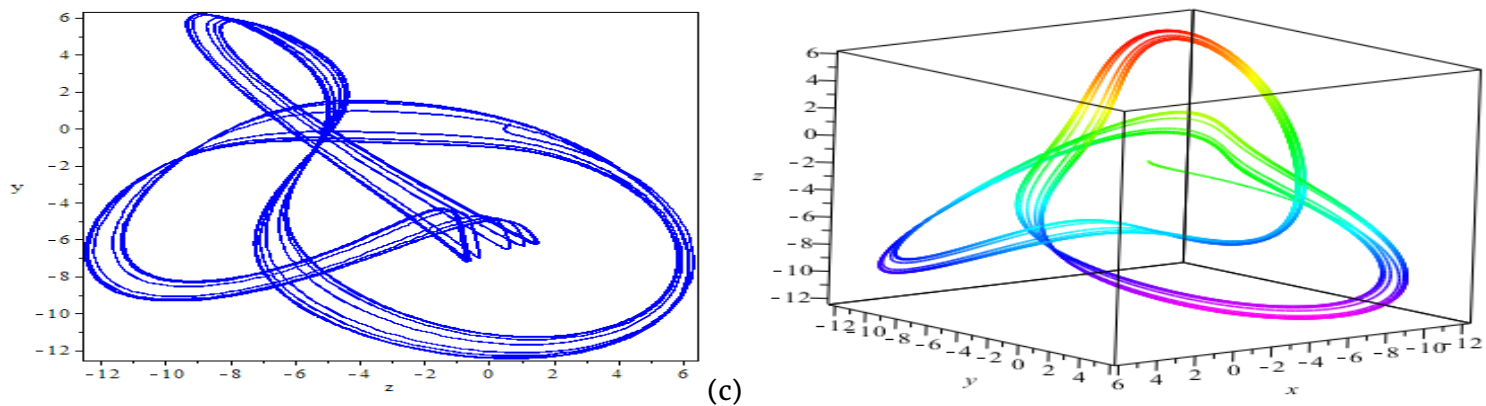

(d)

Figure 5. (a) Phase plane $x-y$, (b) Phase plane $x-z$, (c) Phase plane $y-z$ and (d) The attractor $x-y-z$
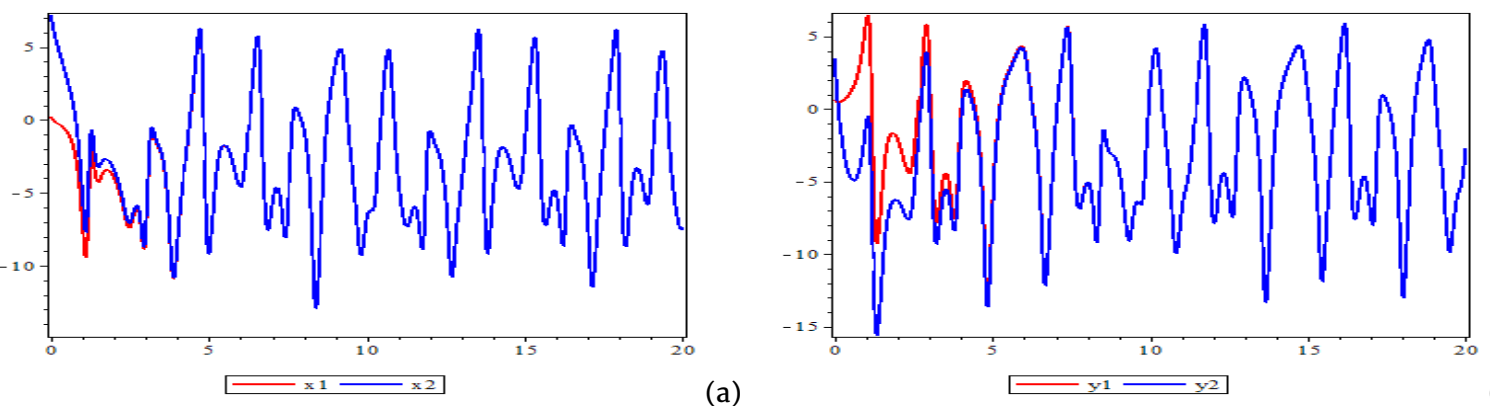

(a)

(b)
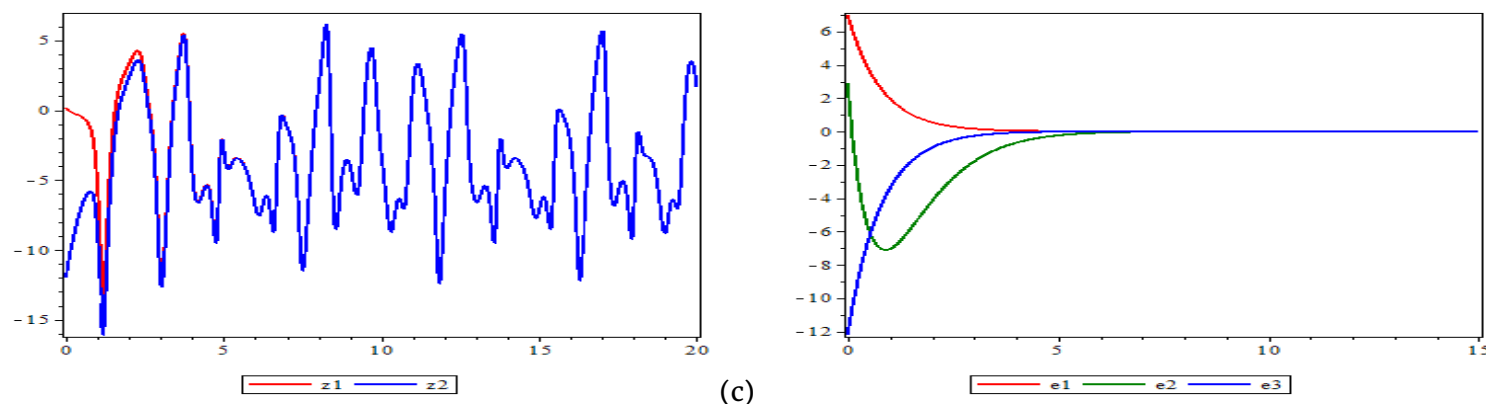

(d)

Figure 6. Synchronization of the VOF system (18): (a) $x_{1}(t)$ vs $x_{2}(t),(\mathrm{b}) y_{1}(t)$ vs $y_{2}(t),(\mathrm{c}) z_{1}(t)$ vs $z_{2}(t)$ and (d) The error functions $e_{1}(t), e 2(t), e 3(t) \rightarrow 0$ as $t \rightarrow \infty$.

\section{Conclusion}

This paper introduced a novel numerical method for solving ordinary differential equations with variable-order time-fractional derivatives. It is shown that there is no computational complexity in the algorithm, the method is easy to program. The accuracy of the method is demonstrated through numerical examples. Moreover, the chaotic dynamics of a Halvorsen system with variableorder fractional derivatives are investigated and the identical synchronization between two systems is achieved. Besides, the results of this paper reveal that the variable-order derivation can be very useful for describing chaotic phenomena, their control, and synchronization. 


\section{Declarations}

\section{Consent for publication}

Not applicable.

\section{Conflicts of interest}

The authors declare that they have no conflict of interests.

\section{Funding}

Not applicable.

\section{Author's contributions}

Z.H.: Conceptualization, Methodology, Software, Writing-Original draft preparation. M.Y.: Data Curation, Validation, WritingReviewing and Editing. N.Ö.: Investigation, Visualization, Supervision. All authors discussed the results and contributed to the final manuscript.

\section{Acknowledgements}

The authors are very much thankful to the reviewers and editor for their valuable suggestions to improve the quality of the manuscript.

\section{References}

[1] Lorenz, E.N. Deterministic nonperiodic flow. Journal of atmospheric sciences, 20(2), 130-141, (1963).

[2] Azar, A.T., Sundarapandian, V. Chaos modeling and control systems design (Vol. 581). Germany: Springer, (2015).

[3] Azar, A.T., Sundarapandian, V., Ouannas, A. Fractional order control and synchronization of chaotic systems (Vol. 688). Springer, (2017).

[4] Effati, S., Saberi-Nadjafi, J., Saberi Nik, H. Optimal and adaptive control for a kind of 3D chaotic and 4D hyper-chaotic systems. Applied Mathematical Modelling, 38(2), 759-774, (2014).

[5] Yang, J., Dong-Lian, Q. The feedback control of fractional order unified chaotic system.Chinese Physics B, 19(2), 020508, (2010).

[6] Li, C., Tong, Y. Adaptive control and synchronization of a fractional-order chaotic system.Pramana, 80(4), 583-592, (2013).

[7] Li, C.G., Chen, G.R. Chaos in the fractional order Chen system and its control. Chaos, Solitons and Fractals, 22(3), 549-554, (2004).

[8] Li, Y., Chen, Y., Podlubny, I. Stability of fractional-order nonlinear dynamic systems: Lyapunov direct method and generalized Mittag-Leffler stability. Computers and Mathematics with Applications, 59(5), 1810-1821, (2010).

[9] Matignon, D. Stability results for fractional differential equations with applications to control processing.In Computational Engineering in Systems Applications, 963-968, (1996).

[10] Mekkaoui, T., Hammouch, Z., Belgacem, F., Abbassi, A.E. Fractional-order nonlinear systems: Chaotic dynamics, numerical simulation and circuits design. In Fractional Dynamics, (pp.343-356). Sciendo Migraiton, (2016).

[11] Auerbach, D., Grebogi, C., Ott, E., Yorke, J.A. Controlling chaos in high dimensional systems. Physical review letters, 69(24), 3479, (1992).

[12] Pyragas, V., Pyragas, K. Continuous pole placement method for time-delayed feedback controlled systems. The European Physical Journal B, 87(11), 1-10, (2014).

[13] Razminia, A., Baleanu, D. Fractional synchronization of chaotic systems with different orders.Proceedings of the Romanian Academy, 13, 14-321, (2012).

[14] Bai, E.W., Lonngren, K.E. Synchronization of two Lorenz systems using active control. Chaos, Solitons Fractals, 8(1), 51-58, (1997).

[15] Blokh, A., Cleveland, C., Misiurewicz, M. Expanding polymodials Modern Dynamical Systems and Applications ed M Brin, B Hasselblatt and Ya Pesin, 253-70, (2004).

[16] Chamgoué, A., Yamapi, R., Woafo, P. Bifurcations in a birhythmic biological system with time-delayed noise. Nonlinear Dynamics, 73(4), 2157-2173, (2013).

[17] Diethelm, K., Ford, N. Analysis of fractional differential equations. Journal of Mathematical Analysis and Applications, 265(2), 229-248, (2002).

[18] Diethelm, K., Ford, N., Freed, A., Luchko, Y. Algorithms for the fractional calculus: a selection of numerical method. Computer Methods in Applied Mechanics and Engineering, 194(6-8), 743-773, (2005).

[19] Frohlich, H. Long Range Coherence and energy storage in a Biological systems. International Journal of Quantum Chemistry, 2(5), 641-649, (1968).

[20] Frohlich, H. Quantum Mechanical Concepts in Biology. Theoretical Physics and Biology, 1, (1969).

[21] He, G., Luo, M. Dynamic behavior of fractional order Duffing chaotic system and its synchronization via singly active control. Applied Mathematics and Mechanics, 33(5), 567-582, (2012). 
[22] Kadji, H.G., Orou, J.B., Yamapi, R., Woafo, P. Nonlinear Dynamics and Strange Attractors in the Biological System. Chaos Solitons and Fractals, 32(2), 862-882, (2007).

[23] Kaiser, F. Coherent Oscillations in Biological Systems I. Bifurcations Phenomena and Phase transitions in enzyme-substrate reaction with Ferroelectric behaviour, 294, 304-333, (1978).

[24] Kaiser, F. Coherent Oscillations in Biological Systems II. Lecture Notes in Mathematics 2007; 1907; Springer: Berlin.

[25] Miller, K.S., Rosso, B. An Introduction to the Fractional Calculus and Fractional Differential Equations. Wiley, New York, (1993).

[26] Pham, V.T., Frasca, M., Caponetto, R., Hoang, T.M., Fortuna, L. Control and synchronization of fractional-order differential equations of phase-locked-loop. Chaotic Modeling and Simulation, 4, 623-631, (2012).

[27] Pecora, L.M., Carroll, T.L. Synchronization in chaotic systems. Phys Rev Lett, 64, 821-824, (1990).

[28] Pikovsky, A. Synchronization: A Universal Concept in Nonlinear Sciences. Cambridge University Press, (2011).

[29] Strogatz, S.H. Nonlinear Dynamics and Chaos: With Applications to Physics, Biology, Chemistry and Engineering. Perseus Books Pub, (1994).

[30] Ucar, A., Lonngren, K.E., Bai, E.W. Synchronization of the unified chaotic systems via active control. Chaos, Solitons and Fractals, 27,1292-97, (2006).

[31] Zaslavsky, G. Hamiltonian Chaos and Fractional Dynamics. Oxford University Press, (2008).

[32] Katsikadelis, J.T. Numerical solution of variable order fractional differential equations.arXiv preprint arXiv, 1802.00519, (2018).

[33] Podlubny, I. Fractional Differential Equations. Academic Press: San Diego; Calif, USA, (1999).

[34] Erturk, V.S, Momani, S., Odibat, Z. Application of generalized differential transform method to multi-order fractional differential equations. Communications in Nonlinear Science and Numerical Simulation, 13(8), 1642-1654, (2008).

[35] Freihat, A., Momani, S. Application of Multistep Generalized Differential Transform Method for the Solutions of the Fractional-Order Chua's System. Discrete Dynamics in Nature and Society, 1-12, (2012).

[36] Hammouch, Z., Mekkaoui, T. Circuit design and simulation for the fractional-order chaotic behavior in a new dynamical system. Complex and Intelligent Systems, 4(4), 251-260, (2018).

[37] Hongwu, W., Junhai, M. Chaos Control and Synchronization of a Fractional-order Autonomous System. WSEAS Trans. on Mathematics, 11,700-711, (2012).

[38] Caponetto, R., Dongola, G., Fortuna, L. Fractional order systems: Modeling and control application. Singapore: World Scientific, (2010).

[39] Escalante-Martínez, J.E., Gómez-Aguilar, J.F., Calderón-Ramón, C., Aguilar-Meléndez, A. \& Padilla-Longoria, P. A mathematical model of circadian rhythms synchronization using fractional differential equations system of coupled van der Pol oscillators.International Journal of Biomathematics, 11(01), 1850014, (2018).

[40] Coronel-Escamilla, A., Gómez-Aguilar, J.F., Torres, L., Escobar-Jiménez, R.F. \& Valtierra-Rodríguez, M. Synchronization of chaotic systems involving fractional operators of Liouville-Caputo type with variable-order. Physica A. Statistical Mechanics and its Applications, 487, 1-21, (2017).

[41] Yavuz, M. European option pricing models described by fractional operators with classical and generalized Mittag-Leffler kernels. Numerical Methods for Partial Differential Equations, 10.1002/num.22645, (2021).

[42] Zúniga-Aguilar, C.J., Gómez-Aguilar, J.F., Escobar-Jiménez, R.F. \& Romero-Ugalde, H.M. Robust control for fractional variable-order chaotic systems with non-singular kernel. The European Physical Journal Plus, 133(1), 1-13, (2018).

[43] Yavuz, M. Novel solution methods for initial boundary value problems of fractional order with conformable differentiation. An International Journal of Optimization and Control: Theories \& Applications (IJOCTA), 8(1), 1-7, (2018).

[44] Zúniga-Aguilar, C.J., Romero-Ugalde, H.M., Gómez-Aguilar, J.F., Escobar-Jiménez, R.F. \& Valtierra-Rodríguez, M. Solving fractional differential equations of variable-order involving operators with Mittag-Leffler kernel using artificial neural networks. Chaos, Solitons \& Fractals, 103, 382-403, (2017).

[45] Petras, I. A note on the fractional-order Chua's system. Chaos Soliton and Fractals, 38(1), 140-147, (2008).

[46] Petras, I. Fractional-Order Nonlinear Systems: Modeling, Analysis and Simulation. Springer, (2011).

[47] Caputo, M. Linear models of dissipation whose $Q$ is almost frequency independent. Geophysical Journal International, 13(5), 529-539, (1967).

[48] Ucar, S., Ucar, E., Ozdemir, N., Hammouch, Z. Mathematical analysis and numerical simulation for a smoking model with Atangana-Baleanu derivative. Chaos, Solitons and Fractals, 118, 300-306, (2019).

[49] Chand, M., Hammouch, Z., Asamoa, J.K.K., Baleanu, D. Certain Fractional Integrals and Solutions of Fractional Kinetic Equations Involving the Product of S-Function. In Mathematical Methods in Engineering (pp. 213-244), Springer, Cham, (2019).

[50] Owolabi, K.M., Hammouch, Z. Mathematical modeling and analysis of two-variable system with noninteger-order derivative. Chaos: An Interdisciplinary Journal of Nonlinear Science, 29(1), 013145, (2019).

[51] Yavuz, M., Ozdemir, N. European vanilla option pricing model of fractional order without singular kernel. Fractal and Fractional, 2(1), 3, (2018).

[52] Yavuz, M., Ozdemir, N. On the solutions of fractional Cauchy problem featuring conformable derivative.In ITM Web of Conferences. 22, EDP Sciences, (2018).

[53] Yavuz, M., Ozdemir, N. Numerical inverse Laplace homotopy technique for fractional heat equations. Thermal Science, 22(1), 185-194, (2018).

[54] Toufik, M., Atangana, A. New numerical approximation of fractional derivative with non-local and non-singular kernel: Application to chaotic models. The European Physical Journal Plus, 132(10), 1-16, (2017).

[55] Naik, P.A., Yavuz, M., Qureshi, S., Zu, J., Townley, S. Modeling and analysis of COVID-19 epidemics with treatment in fractional derivatives using real data from Pakistan. The European Physical Journal Plus, 135(10), 1-42, (2020).

[56] Mirzazadeh, M., Akinyemi, L., Şenol, M., Hosseini, K. A variety of solitons to the sixth-order dispersive $(3+1)$-dimensional nonlinear time-fractional Schrödinger equation with cubic-quintic-septic nonlinearities. Optik, 241, 166318, (2021).

[57] Yavuz, M., Yokus, A. Analytical and numerical approaches to nerve impulse model of fractional-order. Numerical Methods for Partial Differential Equations, 36(6), 1348-1368, (2020). 
[58] Matar, M.M., Abbas, M.I., Alzabut, J., Kaabar, M.K.A., Etemad, S., Rezapour, S. Investigation of the p-Laplacian nonperiodic nonlinear boundary value problem via generalized Caputo fractional derivatives. Advances in Difference Equations, 2021(1), 1-18, (2021).

[59] Kaabar, M.K., Martínez, F., Gómez-Aguilar, J.F., Ghanbari, B., Kaplan, M., Günerhan, H. New approximate analytical solutions for the nonlinear fractional Schrödinger equation with second-order spatio-temporal dispersion via double Laplace transform method. Mathematical Methods in the Applied Sciences, 44(14), 11138-11156, (2021).

[60] Yavuz, M., Sulaiman, T.A., Usta, F., Bulut, H. Analysis and numerical computations of the fractional regularized long-wave equation with damping term. Mathematical Methods in the Applied Sciences, 44(9), 7538-7555, (2021).

[61] Tariq, K.U., Younis, M., Rizvi, S.T.R., Bulut, H. M-truncated fractional optical solitons and other periodic wave structures with Schrödinger-Hirota equation. Modern Physics Letters B, 34(supp01), 2050427, (2020).

[62] Yaghoobi, S., Moghaddam, B.P., Ivaz, K. An efficient cubic spline approximation for variable-order fractional differential equations with time delay. Nonlinear Dynamics, 87(2), 815-826, (2017).

[63] Coronel-Escamilla, A., Gómez-Aguilar, J.F., Torres, L., Valtierra-Rodriguez, M. \& Escobar-Jiménez, R.F. Design of a state observer to approximate signals by using the concept of fractional variable-order derivative. Digital Signal Processing, 69, 127-139, (2017).

[64] Gómez-Aguilar, J.F. Analytical and numerical solutions of a nonlinear alcoholism model via variable-order fractional differential equations. Physica A: Statistical Mechanics and its Applications, 494, 52-75, (2018).

[65] Gómez-Aguilar J.F. Chaos in a nonlinear Bloch system with Atangana-Baleanu fractional derivatives. Numerical Methods for Partial Differential Equations, 34(5), 1716-1738, (2018).

[66] Yufeng, X., Zhimin, H. Synchronization of variable-order fractional financial system via active control method. Open Physics, 11(6), 824-835, (2013).

[67] Razminia, A., Dizaji, A.F., Majd, V.J. Solution existence for non-autonomous variable-order fractional differential equations. Mathematical and Computer Modelling, 55(3-4), 1106-1117, (2012).

[68] Solís-Pérez, J.E., Gómez-Aguilar, J.F., Atangana, A. Novel numerical method for solving variable-order fractional differential equations with power, exponential and Mittag-Leffler laws.Chaos, Solitons and Fractals, 114, 175-185, (2018).

[69] Atangana, A., Owolabi, K.M. New numerical approach for fractional differential equations. Mathematical Modelling of Natural Phenomena, 13(1), 3, (2018).

[70] Hammouch, Z., Mekkaoui, T. Chaos synchronization of a fractional nonautonomous system. Nonautononmous Dynamical Systems, 1:61-71, (2014)

[71] Lu, L,. Zhang, C., Guo, Z.A. Synchronization between two different chaotic systems with nonlinear feedback control. Chinese Physics, 16, 1603-1607, (2007).

[72] Olusola, O., Vincent, E., Njah, N., Ali, E. Control and Synchronization of Chaos in Biological Systems Via Backsteping Design. International Journal of Nonlinear Science and Numerical Simulation, 11(1), 121-128, (2011).

[73] Haeri, M., Emadzadeh, A. Synchronizing different chaotic systems using active sliding mode control. Chaos, Solitons and Fractals, 31(1), 119-129, (2007).

[74] Wang, Y., Guan, Z.H., Wang, H.O. Feedback an adaptive control for the synchronization of Chen system via a single variable. Physics Letters A, 312(1-2), 34-40, (2003).

Mathematical Modelling and Numerical Simulation with Applications (MMNSA) (http://www.mmnsa.org)

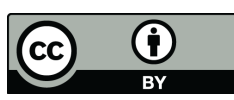

Copyright: () 2021 by the authors. This work is licensed under a Creative Commons Attribution 4.0 (CC BY) International License. The authors retain ownership of the copyright for their article, but they allow anyone to download, reuse, reprint, modify, distribute, and/or copy articles in MMNSA, so long as the original authors and source are credited. To see the complete license contents, please visit (http://creativecommons.org/licenses/by/4.0/). 